\title{
Solutions from nature for building envelope thermoregulation
}

\author{
L. Badarnah, Y. Nachman Farchi \& U. Knaack \\ Facade Research Group, Design of Construction, Building Technology, \\ Faculty of Architecture, Delft University of Technology, The Netherlands
}

\begin{abstract}
The building envelope has to maintain a thermal comfort for the occupants. Current technologies for buildings consider the envelope as a thermal barrier or a shield that has to be insulated to prevent heat loss and allow it to be open to dissipate heat if necessary. More efficient thermoregulation solutions can be found in nature. Organisms can manipulate their body temperature by behavioural or physiological means as an adaptive response to the environmental changes. In this paper we present performance taxonomy of organisms that facilitate thermoregulation in nature, and we discuss their possible application in building envelopes. Moreover, we present an application case of such taxonomy for an evaporative cooling system for building envelopes.
\end{abstract}

Keywords: thermoregulation, building envelope, skin, sweating, cooling, heating, conduction, convection, radiation, evaporation.

\section{Introduction}

One of the aims of the building envelope is to maintain a thermal comfort in the enclosed spaces occupied by people. Ventilation, cooling, heating, and solar radiation significantly influence the thermal comfort of a space. There are various developed technologies that manipulate these aspects by using a great deal of energy and time. In these technologies, the envelope is considered as a thermal barrier or a shield that has to be insulated to prevent heat loss and allow it to be open to dissipate heat if necessary. This way of conception limits more efficient solutions, where the building envelope is not considered as a barrier but as a medium. 
Living organisms have to maintain their body temperature in a very narrow range in order to survive. They can manipulate it by behavioural or physiological manners. In addition to generating heat metabolically, organisms exchange heat with their surroundings by: conduction, convection, evaporation, and thermal radiation [1]. Maintaining a stable body temperature is achieved by a continuous process of heat gain and loss. A wide range of strategies are adapted to facilitate heat gains and losses by organisms. In some organisms, the process is achieved through the skin which functions as a thermal filter, whereas in others, it is achieved by their built structures. Different mechanisms are adapted for different climates and for different species.

Up to now, strategies and mechanisms adapted by organisms are not utilized in designing thermo regulating systems for building envelopes. We analyse thermoregulation in a number of organisms in order to be applied in building envelopes. The advantage of applying such thermoregulation mechanisms is to improve the thermal comfort with less energy demanding operation. As an outcome of the analysis we provide performance taxonomy of organisms that facilitate thermoregulation. Moreover, we present an application case of such taxonomy for an evaporative cooling system (Stoma Brick) for building envelopes.

\section{Thermoregulation in nature}

Thermoregulation is the action of keeping inner temperature levels of an organism within certain boundaries (the balance between heat gains and losses) despite of having changing temperatures in the surrounding environment. Certain ranges of temperatures are required for organisms to perform biological functions. As a result, they have adopted physiological or behavioural methods for thermoregulation [2]. Air temperature, solar radiation, radiation from other objects, absorptivity of the organism, wind speed, and convection can influence body temperature $[3,4]$.

\subsection{Physics of heat transfer}

Beyond making heat metabolically, heat is transferred between animals and their environment by conduction, convection, radiation, and evaporation [5]. The body of an organism is in constant balancing processes between heat gain and heat loss. Nature has different strategies for cold and hot environments.

\subsubsection{In the cold}

In cold environments maintaining a proper temperature for the organism is done basically by reducing heat loss and/or increasing heat gain. Such maintenance is achieved through the following methods [5]:

- Increase in heat production - achieved through muscular activity and exercise, involuntary muscle contractions (shivering), and non-shivering thermogenesis. 
- Conduction, insulation, and fur thickness - the level of heat flow from the body to the environment is called conduction. When conduction is low then insulation is high. Fur is basically for insulation; its thickness for some animals is changing throughout the seasons. For example, in the summer the black bear loses $52 \%$ of the insulation value of its winter fur.

- Conductance in birds - birds decrease conductance in the cold by raising their feathers and withdraw head and feet into the feathers. They also allow the peripheral tissues temperature to drop while marinating the core temperature. This results in a decreased shell circulation, an increased shell thickness, and a reduced core volume. All these contribute in maintaining the core temperature.

- Huddling - penguins in the Antarctic huddle together during the breeding season. It reduces the surface area in contact with the cold air (reducing the cold stress).

- Insulation in aquatic mammals - seals and whales that live and swim in the arctic and Antarctic sea have a thick layer of subcutaneous blubber for insulation, since fur loses most of its insulation value in water. Aquatic mammals modulate heat loss from the skin by blood bypassing the insulator.

- Distribution of insulating material - different body parts are not equally insulated, since animals need surfaces from which heat can be dissipated if needed. Different thicknesses of insulating material over the body surface allow a considerable flexibility in regulating conductance by changes in the posture.

- Heat exchangers - It is achieved due to the special structure of the blood vessels, e.g. the flippers of the whale: each artery is completely surrounded by veins. This special arrangement cools the arterial blood before reaching the periphery and thus loses a little heat to the water, and warms the returning blood (venous blood) before entering the body.

\subsubsection{In the heat}

In environment where the ambient temperature is higher than body temperature, the body receives heat by conduction from the hot air, by radiation from sun, and by the heated ground surface. Evaporation is often used by mammals to dissipate the metabolic heat production and the heat gained from the environment.

- The importance of body size - since heat gain of an object by conduction, convection and radiation processes has a direct relation to surfaces; the sum of environmental heat load on an object is directly related to surface area. A small animal loses heat to the surroundings rapidly and can not maintain his body temperature very different from the medium.

- Evaporation- sweating, panting, and gular fluttering - these processes are basically adapted to increase cooling by evaporation. When air flows over a moist surface it causes evaporation, which in turn takes a certain amount of heat from the surface. Sweating, panting, and gular fluttering are mechanisms that occur in different species. The capability of sweating is found in some mammals including humans, horses, camels, and some kangaroos. However, birds do not sweat, that is why they have adopted gular fluttering which occurs in their mouth - they keep their mouth open and vibrate the floor of the mouth 
(gular area) to increase the airflow and as a result promote evaporation. Panting is common among birds and mammals, where the rate of breathing is increased as a result of heat stress, e.g. dogs [1].

\subsection{Poikilotherms}

Poikilotherms are also called ectotherms, which include amphibians, most fish, most nonavian reptiles, all aquatic invertebrates, and most terrestrial invertebrates. They are characterized by varying body temperature in response to changing thermal conditions of the environment. Their mechanism of thermoregulation is behavioural [5].

Behavioural thermoregulation - is when the behaviour of an organism is changed in response to body temperature changes. Their principal source of body heat is the environment.

\subsection{Hometherms}

Hometherms are also called endotherms, which include mammals and birds. They can acclimatize to changing seasons by acclimatizing one or more of the following: metabolic rate, metabolic endurance, and insulation. endotherms are characterized by regulating their body temperature by physiological means.

Physiological thermoregulation - is when the physiology of an organism is changed for temperature regulation. Their principal source of body heat is generated metabolically.

\section{Models from nature for thermoregulation}

Nature has unique strategies that allow it to adapt to the fluctuating environmental temperatures. Different climates have different conditions and unique species that can tolerate the environmental changes throughout the seasons. The following organisms and built structures from nature facilitate effective strategies/mechanisms for thermoregulation, despite the changes in ambient temperature. A summary of their strategies and main principles are given in section 3.5, where we discuss their possible application in building envelope design for thermoregulation.

\subsection{Termite mounds - passive ventilation}

Nest site selection, orientation, and architecture, play a major role in regulating nest's microclimate [6]. The amount of solar radiation reaching the surfaces of the mound is influenced by the orientation and the shape of the mound [7]. In order to control maximum and minimum exposure throughout the day, mounds have a specific long axis facing [8]. For example, long axis oriented north-south, will increase exposure to solar radiation at early morning and late in the afternoon, and resulting in the less exposure of solar radiation at noon. Termite mounds have some structural features to retain heat and others for dissipating it. 
They include variation in wall thicknesses, mound surface design or projecting structures, and orientation as already mentioned [6]. Mounds in Savannah (warm environment) have thin walls with numerous ridges and turrets (enlarged surface area), whereas mounds in the gallery forest (cooler environment) are domeshaped with thick walls and few projecting structures (reduced surface area). This difference in mound walls results in more heat dissipation in the mounds at Savannah and retain more heat in the forest mounds [9, 10]. A large variety of nest structures are adapted for ventilation: enormous chimneys, air passages close to the surface of mounds without chimneys [11, 12]. Thus, the mound has to find the balance between temperature regulation and ventilation. The reduced surface area in the forest limits gas exchange through the mound, and happens mostly at the crest of the mound [12]. At warmer environments (Savannah), the surface area is not affected by increasing temperatures. Thus gas exchange occurs through holes all over the surfaces of the mound.

Heat is also generated metabolically, and by clustering together (the termites) they keep a stable temperature inside the nest [13]. The architecture of the nest is dynamic and adaptive; the inhabitants modify it for a better performance in accordance to the environmental changes [14].

\subsection{Tuna fish - heat exchange}

Tunas have vascular counter-current heat exchangers that maintain their body temperature above the ambient water temperature, e.g. the muscle temperature of the bluefin tuna is $20^{\circ} \mathrm{C}$ above the ambient water temperature [15]. That is why they are considered as unique and extraordinary among fish in thermoregulation aspects [16]. Tunas have a highly developed system of counter-current heat exchangers within the vascular system of the muscle acting as a barrier preventing heat loss through the gills. By cooling the surface, the thermal gradient (surface, body, and water) is reduced, resulting in improved heat conservation [17].

Tuna species have central and lateral heat exchangers, which are different in their sizes among species [18]. For example, the central heat exchanger in the skipjack tuna is more advanced than the lateral one, the yellow fin tuna has advanced central and lateral heat exchangers, and the bigeye tuna has an advanced lateral heat exchanger [19].

The blood vessels that supply the major active muscles in the tuna run along the edge under the skin. They are arranged in rete mirabile. From the major vessels branch many parallel fine blood vessels forming a slab, where the arteries are interspersed with the veins (running in opposite directions). Heat exchange is assisted by the small diameter (about $0.1 \mathrm{~mm}$ ) of the vessels [5].

\subsection{Human skin - sweating}

Heat exchange mechanisms in human body include: sensible heat transfer at skin surface, latent heat transfer, and sensible and latent exchange via respiration from the lungs. 
The skin provides a medium between the organism and the surrounding environment, which has to control heat and moisture transfer in response to thermoregulatory demands of the body. It contains cold and warm sensors, where cold sensors are closer to the surface than warm sensors [20], and ten times more in amount than the warm sensors [21]. This organisation allows a rapid detection of cold than warmth [22], which suggests that humans are more sensitive to danger from cold than from heat.

Despite thickness variations of the skin, in general it is about $2 \mathrm{~mm}$ thick, containing a complex vascular system and sweat glands. It includes two main layers: the epidermis and the dermis. The epidermis is the outermost layer of the skin, and the primary barrier to water diffusion [23]. The dermis is much thicker than the epidermis and containing vascular systems, sweat glands, and thermoregulatory nerves [24]. A subcutaneous or fat layer lies beneath the dermis which functions as insulation and stored food energy. It is about 17 times thicker than the dermis and has different thicknesses among individuals [25].

Thermoregulation by vasoconstriction or vasodilatation reduces or enhances (respectively) blood circulation to the skin surface. This process promotes heat loss by conduction to the surrounding with vasodilatation, and prevents heat loss in the cold with vasoconstriction. A dense vascular network (the venous plexus) in the subcutaneous region has a major effect on skin temperature and heat transfer from the skin to the surrounding environment. When arterio-venous anastamoses (valves) are open, they provide a shortcut for the blood route from the arterioles to the venous plexus, which act as a warm chamber next to skin surface, resulting in heat loss via conduction to the environment.

When sensible heat transfer becomes insufficient for heat removal, then moisture evaporation (latent heat transfer) becomes an efficient heat removal process. Eccrine sweat glands through their openings on the skin surface provide moisture on the skin. Increasing the secreted sweat to the skin surface is achieved by both increasing the number of contributing sweat glands and by increasing the amount of output of each active glands. An increase in the output of each gland was observed as the primary result of overheating, rather than increasing the number of participating sweat glands [26, 27].

\subsection{Birds - gular fluttering}

Gular fluttering is a process adapted by some species of birds in order to increase the rate of evaporative cooling. In this process the bird increases air flow over the moist vascular oral membranes by vibration; this in turn increases evaporation and results in increased dissipated heat. Increased heat load results in increased gular fluttering [28].

\subsection{Taxonomy for possible application in building envelopes}

This section provides an overview on the explored natural systems that perform homeostasis as summarized in table 1 . In table 1 we recognize active and passive mechanisms adapted by the organisms. Different physiological features of organisms require different strategies. Organisms living in different habitats have unique mechanisms to perform thermoregulation. 
Organisms have to maintain homeostasis for survival. Similarly, the building should maintain thermal comfort. Designers often seek air-tightness and watertightness to improve the thermal performance of buildings. However, organisms succeed to maintain an adequate balance between heat gain and heat loss without seeking air-tightness and water-tightness.

Table 1: $\quad$ Performance taxonomy of the organisms.

\begin{tabular}{|c|c|c|c|c|}
\hline Organism & Termite mound & Tuna fish & Human skin & Birds \\
\hline Main feature & Passive ventilation & Heat exchange & $\begin{array}{c}\text { Sweating and } \\
\text { vasoconstriction / } \\
\text { vasodilatation }\end{array}$ & $\begin{array}{c}\text { Gular } \\
\text { fluttering }\end{array}$ \\
\hline Challenge & $\begin{array}{c}\text { Maintain stable } \\
\text { temperatures inside } \\
\text { the mound throughout } \\
\text { the day }\end{array}$ & $\begin{array}{c}\text { Conserve heat in cold } \\
\text { water }\end{array}$ & $\begin{array}{c}\text { Body } \\
\text { thermoregulation }\end{array}$ & Cooling \\
\hline Strategy & $\begin{array}{c}\text { The inhabitants of the } \\
\text { mound modify it in } \\
\text { accordance to the } \\
\text { environmental } \\
\text { changes }\end{array}$ & \begin{tabular}{|} 
Reducing thermal \\
gradient (body- \\
environment) to \\
improve heat \\
conservation. This is \\
done by lateral heat \\
exchangers
\end{tabular} & $\begin{array}{c}\text { Contains a complex } \\
\text { vascular system and } \\
\text { sweat glands for } \\
\text { evaporative cooling }\end{array}$ & $\begin{array}{c}\text { Increasing } \\
\text { the rate of } \\
\text { evaporative } \\
\text { cooling }\end{array}$ \\
\hline Mechanism & \begin{tabular}{|c|} 
Structural features to \\
retain or dissipate \\
heat: variations in \\
wall thicknesses, \\
surface design, \\
projecting structures, \\
orientation, chimneys, \\
air passages, porosity
\end{tabular} & \begin{tabular}{|} 
- Blood vessels that \\
supply the active \\
muscles run under \\
the skin and \\
arranged in rete \\
mirabile. \\
- The small diameter \\
of the vessels assist \\
heat exchange
\end{tabular} & \begin{tabular}{|c|} 
- Enhancing or \\
reducing blood \\
circulation to the \\
skin surface affects \\
heat loss via \\
conduction. \\
- Secreting sweat to \\
the skin surface \\
removes heat by \\
evaporation. \\
\end{tabular} & \begin{tabular}{|c|} 
Increasing \\
airflow over \\
the moist \\
vascular oral \\
membranes \\
via rapid \\
vibration
\end{tabular} \\
\hline Active/ Passive & passive & passive & active/passive & active \\
\hline $\begin{array}{c}\text { Behavioural/ } \\
\text { Morphological/ } \\
\text { Physiological } \\
\end{array}$ & Physiological & $\begin{array}{l}\text { Physiological } \\
\text { Morphological }\end{array}$ & Physiological & Physiological \\
\hline Main principle & $\begin{array}{l}\text { Chimneys and air } \\
\text { passages for passive } \\
\text { ventilation }\end{array}$ & $\begin{array}{l}\text { Blood vessels } \\
\text { arranged in rete } \\
\text { mirabile. } \\
\text { Lateral counter- } \\
\text { current heat } \\
\text { exchanger }\end{array}$ & $\begin{array}{l}\text { Latent heat transfer } \\
\text { for cooling. } \\
\text { Heat loss by } \\
\text { conduction. }\end{array}$ & $\begin{array}{l}\text { Dissipate } \\
\text { heat via } \\
\text { vibration }\end{array}$ \\
\hline $\begin{array}{l}\text { Biomimicry } \\
\text { taxonomy* }\end{array}$ & \multicolumn{4}{|c|}{$\begin{array}{l}\text { Group: maintain physical integrity } \\
\text { Sub-group: regulate physical processes } \\
\text { Function: homeostasis }\end{array}$} \\
\hline
\end{tabular}

*AskNature.org - The Biomimicry Institute.

Organisms can adapt to conflicting conditions by adjusting their system behaviour (e.g. vasoconstriction / vasodilatation in human skin). This principle of multi-functionality reduces energy consumption for such organism. Based on the main principles for thermoregulation in nature, we are analogously able to transform these principles and methods into technical solutions for building envelope thermoregulation. 


\section{Current technologies used for building thermoregulation}

The increasing demands concerning comfort resulted in various technologies for ventilating, heating, cooling, acoustic comfort, and visual comfort. Ventilation methods do not conserve heat and result in room temperature drop, which requires a high energy demand to maintain the required temperature. The emerging trend of adaptive facades has developed a wide range of facades that has the ability to adapt to different environmental conditions, such as: collector facades, double facades, alternating facades, and integrated facade [30].

The standard technologies for heating are radiators and heating surfaces. They are attached, in general, under the windows where the highest heat loss occurs, or integrated in the floor or the ceiling [31]. Another heating method is by warm water running through the facade and radiates the heat into the space. It is possible to cool the water in warm seasons [30]. For cooling there are the centralized/decentralized systems distributed and installed in different parts of the building. A wide range of insulating materials is available in the market to influence the conductivity of the exterior wall (envelope). Lately, phase-change materials are applied for better thermal performance of the building envelope.

Current technologies main aim is to maintain a thermal balance in the enclosed spaces, where they use a great deal of energy and time in order to manipulate temperature differences. In these technologies, the envelope is considered as thermal barrier or shield that has to be insulated to prevent heat loss and allow it to be open to dissipate heat if necessary. This way of conception limits more efficient solutions, where the building envelope is not considered as a barrier but as a medium.

\section{Stoma brick}

In this section we present an application of the taxonomy methodology for arid and dry climates. An evaporative cooling system (Stoma Brick - SB) for building envelopes (fig. 1) was designed based on principles (Table 2) of several natural systems. These include stoma of a plant, pine cones, hair protecting eyes in the desert, and human skin.

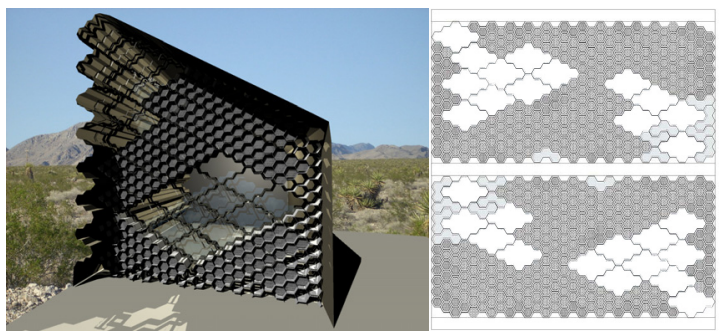

Figure 1: Left: an outer view of the facade. Right: the two different configurations for different organisations, 3 (light gray) or 9 (white) SBs. 
The system consists of four integrated parts (fig. 2):

1. The Stoma brick - SB (fig. 4): made of porous material, which is the functional part for thermoregulation. It has an outer layer of hairy structure to filter the air passing through the envelope. A veneer shutter to control opening/closing in accordance to humidity gradient. The most inner layer is spongy to hold moisture for evaporation.

2. The mono-brick: it includes an irrigation cycle that irrigates through holes the SBs (fig. 5), which are inserted into the mono-brick to allow a continuous performance vertically. Two configurations of mono-bricks sexist for this envelope, $3 \mathrm{SBs}$ and $9 \mathrm{SBs}$, depend on their position in the specific envelope design.

3. The steal framing: is the load bearing structure of the cooling system.

4. The inner layer: HEPA filter for air cleaning or a double acrylic glass for lightening and visual contact with the exterior environment.

Table 2: $\quad$ Summary of the deep principles used for the SB design.

\begin{tabular}{|c|c|}
\hline Natural system & Deep principle \\
\hline Stoma of plants & Osmotic pressure changes control openings for evaporation \\
\hline Pine cone & Relative humidity changes cause material deformation \\
\hline Hair around eyes & Protection against small particles (e.g. dust and sand) \\
\hline Human skin & Latent heat transfer - Cooling trough evaporation \\
\hline
\end{tabular}

The system operates at hot and humid weather: the veneer shutter deforms when humidified, this allows the air to get inside passing through the spongy structure. At hot and dry weather the system functions differently: the irrigating cycles are activated letting water droplets to fall on the veneer shutter in order to cause a deformation and open it allowing the air to enter and pass through the humidified spongy structure. As a result, the dry air that entered is humid when it reaches the inner space.

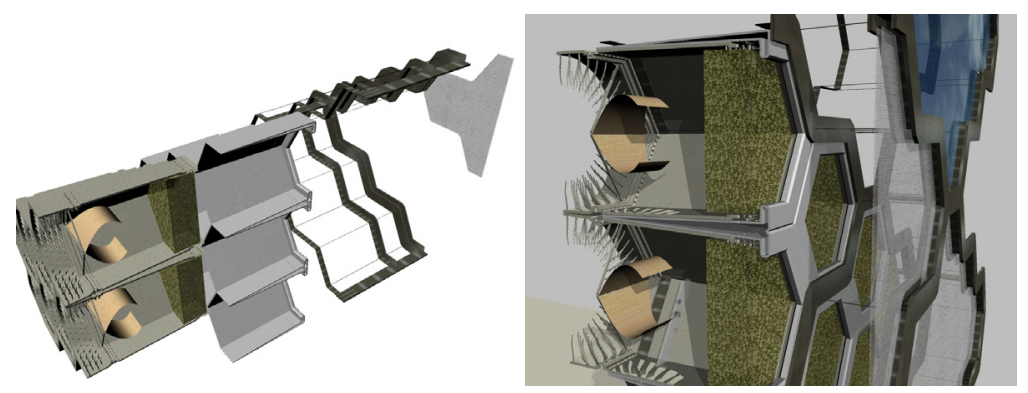

Figure 2: Left: the four integrated parts shown separately. Right: the parts are attached to each other creating one system.

In cold and dry weather, the spongy structure acts as an insulating layer reducing heat loss. Hot and exhausted air is driven outside by upper blocks close to the ceiling. A rapid prototype of the SB is presented in fig. 3. 


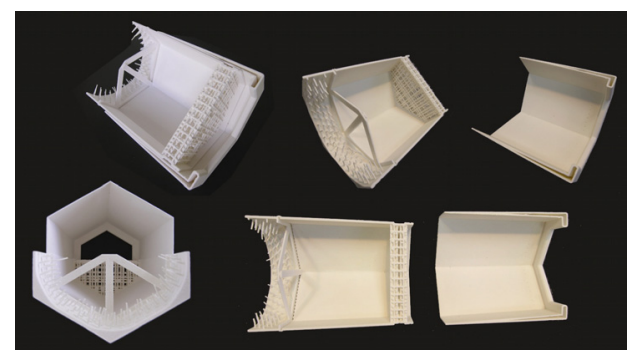

Figure 3: A rapid prototype of the stoma brick.
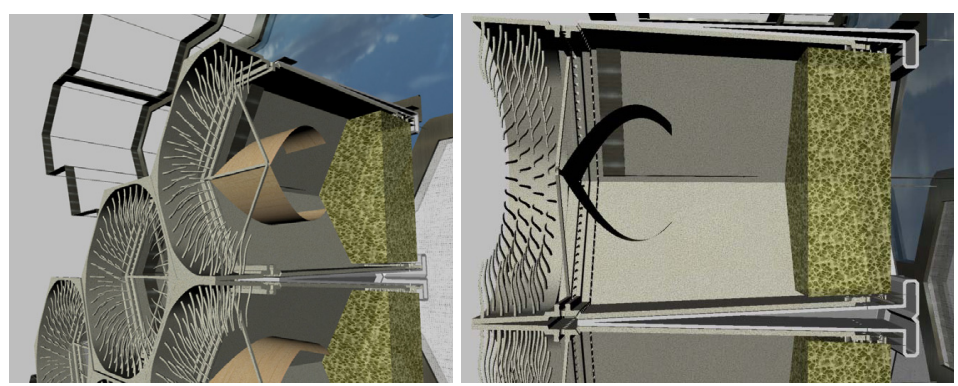

Figure 4: A cross section through the SB showing the deformed veneer (as a reaction to humid environment) allowing the air to enter and ventilate the inner space.
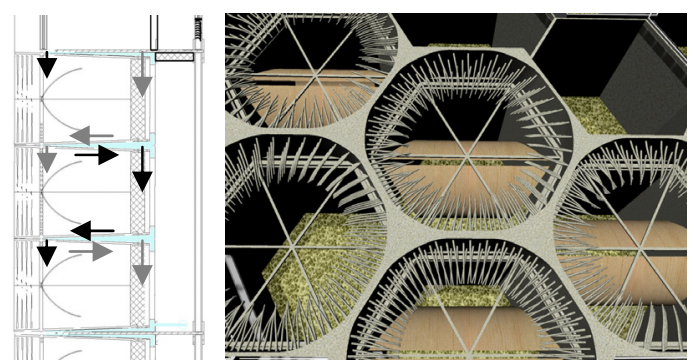

Figure 5: An integrated irrigation system to supply moisture. Two irrigating cycles (black and gray arrows) are active when operated.

\section{Conclusions}

Performance taxonomy of organisms that facilitate thermoregulation is presented for a possible application in building envelopes. This taxonomy is meant to abstract biological processes for non-biologists by main principles extraction. Different taxonomies can be obtained from reviewing biological literature. This way of classification provides the designer brief information for the design challenge. 
Organisms based on their habitats and physiological characteristics adopt different strategies (active or passive), they perform thermoregulation by physiological, behavioural, or morphological means. A significant aspect is their local adaptation: their body parts respond differently to a change in the environment. Organisms succeed to maintain an adequate balance between heat gain and heat loss without seeking air-tightness and water-tightness. Such adaptation solutions by organisms could be applied in buildings with similar challenges. A design case based on solutions adapted by organisms is presented. The design case shows the possible realization of a cooling system. It has an advantage of adapting to changing humidity levels passively.

The transformation of strategies available in nature into technical solutions for building envelopes is a complicated multidisciplinary process. It becomes even more complicated when integrating a number of strategies from different organisms to achieve improved solutions, e.g. an envelope that exchanges heat like Tuna, regulates heat loss by vasoconstriction/ vasodilatation as the skin does, and ventilating by gular fluttering.

\section{References}

[1] Hill, R.W., Wyse, G.A. \& Andreson, M., Animal Physiology, second edition, Sinauer Associates, Inc.: Massachusetts, pp. 205-252, 2008.

[2] Kipervaser, D., Behavioral thermoregulation in terrestrial arthropods, http://www.biologybrowser.org/node/1162349, 2003.

[3] Stevenson, R. D., The Relative Importance of Behavioral and Physiological Adjustments Controlling Body-Temperature in Terrestrial Ectotherms. American Naturalist, 126, pp. 362-386, 1985.

[4] Tracy, C. R. \& Christian, K. A., Ecological Relations among Space, Time, and Thermal Niche Axes. Ecology, 67, pp. 609-615, 1986.

[5] Schmidt-Nielsen, K., Animal physiology: adaptation and environment, Cambridge University Press: New York, pp. 217-297, 2007.

[6] Jones, J.C. \& Oldroyd, B.P., Nest Thermoregulation in Social Insects. Advances in Insect Physiology, 33, pp. 153-191, 2006.

[7] Hubbard, M. \& Cunningham, W., Orientation of mounds of the ant Solensopsis invicta (Hymenoptera, Formicidae, Myrmicinae). Insectes Sociaux, 24(1), pp. 3-7, 1977.

[8] Cole, B.J. Nest architecture in the western harvester ant, Pogonomyrmex occidentalis (Cresson). Insectes Sociaux, 41, pp. 401-410, 1994.

[9] Korb, J. \& Linsenmair, K. E., The effects of temperature on the architecture and distribution of Macrotermes bellicosus (Isoptera, Macrotermitinae) mounds in different habitats of a West African Guinea savanna. Insectes Sociaux, 45(1), pp. 51-65, 1998.

[10] Korb, J. \& Linsenmair, K. E., The architecture of termite mounds: a result of a trade-off between thermoregulation and gas exchange?. Behavioral Ecology, 10(3), pp. 312-316, 1999.

[11] Luscher, M., Air-conditioned termite nests. Scientific American, 205, pp.138-145, 1961. 
[12] Korb, J. \& Linsenmair, K. E., Ventilation of termite mounds: new results require a new model. Behavioral Ecology, 11(5), pp. 486-494, 2000.

[13] Greaves, T. Temperature studies of termite colonies in living trees. Australian Journal of Zoology, 12, pp. 250-262, 1964.

[14] Turner, J.S., The Extended Organism: The Physiology of Animal-Built Structures, Harvard University Press: Cambridge, 2002.

[15] Don Stevens, E., Vascular anatomy of the counter-current heat exchanger of skipjack tuna. Experimental Biology, 61(1), pp. 145-153, 1974.

[16] Holland, K.M., Brill, R.W., Chang, R.K.C., Sibert, J.R., \& Fournier, D.A, Physiological and behavioural thermoregulation in bigeye tuna (Thunnus obesus). Nature, 358(6385), pp. 410-412, 1992.

[17] Carey, F.G. \& Teal, J.M., Heat conservation in tuna fish muscle. Zoology, 56, pp. 1464-1469, 1966.

[18] Brill, R.W., Dewar, H. \& Graham, J.B., Basic concepts relevant to heat transfer in fishes, and their use in measuring the physiological thermoregulatory abilities of tunas. Environmental Biology of Fishes, 40, pp. 109-124, 1994.

[19] Godsil, H.C. \& Bayers, R.D. A systematic study of the Pacific tunas. Calif. Fish. Bull. 60, pp. 1-131, 1944.

[20] Hensel, H., Thermoreception and Temperature Regulation. Academic Press: London, 1982.

[21] Guyton, A.C. \& Hall, J.E., Textbook of Medical Physiology, W.B. Saunders Company: Philadelphia, 2000.

[22] Arens, E.A. \& Zhang, H., The Skin's Role in Human Thermoregulation and Comfort (Chapter 16). Thermal and Moisture Transport in Fibrous Materials, eds. N. Pan \& P. Gibson, Woodhead Publishing Ltd: Cambridge, pp. 560-602, 2006.

[23] Forslind, B. \& Lindberg, M., Skin, Hair, and Nails: Structure and Function, Marcel Dekker: Basel, 2004.

[24] Rushmer, R.F., Buettner, K.J.K., Short, J.M. \& Odland, G.F., The skin. Science, 154, pp. 343-348, 1966.

[25] Stolwijk, J.A.J. \& Hardy, J.D., Skin and subcutaneous temperature changes during exposure to intense thermal radiation. Journal of Applied Physiology, 20, pp. 1006-1013, 1965.

[26] Randall, W.C., Quantitation and regional distribution of sweat glands in man. Clinical Investigation, 25(5), pp. 761-767, 1946.

[27] Randall, W.C., Local sweat gland activity due to direct effects of radiant heat. American Journal of Physiology, 150, pp. 365-371, 1947.

[28] Bartholomew, G.A., Lasiewski, R.C. \& Crawford, Jr., E.C., Patterns of panting and gular flutter in cormorants, pelicans, owls and doves. The Condor, 70, pp. 31-34, 1968.

[29] AskNature, http://www.asknature.org/

[30] Knaack, U., Tillmann, K., Bilow, M. \& Auer, T., Facades: Principles of Construction, BIRKHAUSER: Berlin, 2007.

[31] Daniels, K., Advanced Building Systems: A technical Guide for Architects and Engineers, BIRKHAUSER: Basel, 2003. 